\section{Die Zukunft liegt im richtigen Therapiemix}

$\mathrm{D}$ ie Veränderungen im Gesundheitswesen führen zu einem Arbeitsaufkommen des Allergologen von mindestens elf Stunden pro Tag, das zudem stark saisonal geprägt ist. So bleibt trotz hoher Verantwortung wenig Zeit, um die Patienten optimal zu betreuen. Vor diesem Hintergrund hat Dr. Andreas Horn, Heidelberg, darüber nachgedacht, was ein moderner Therapiemix in der allergologischen Praxis beinhalten könnte. Im Zentrum steht für ihn die spezifische Immuntherapie (SIT) als die einzige kausale Therapie, die den Patienten vor einem Etagenwechsel und Neusensibilisierungen schütze. Der subkutanen Injek-

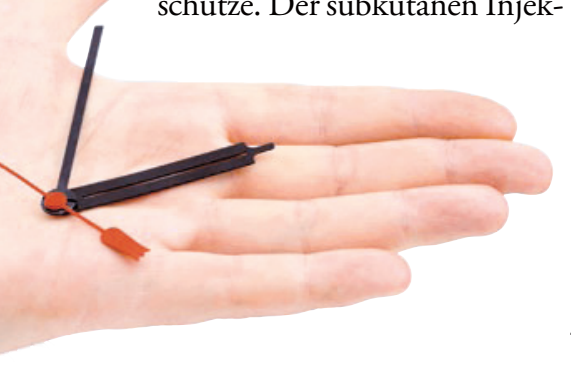

Die Behandlungszeit ist durch planvolle Therapiewahl in den Griff zu bekommen. tion (SCIT) wird die sublinguale Gabe (SLIT) als Tropfen oder Tabletten in der gültigen Leitlinie gleichgestellt. Zur Wahl der Verabreichungsform empfahl Horn: „Man muss den Patienten mit ins Boot nehmen. Denn nur dann bekommen wir auch eine hohe Compliance." Hält der Patient die dreijährige Therapie durch, sei dies auch kostengünstiger, als wenn er vorzeitig abbricht.

Um die saisonalen Peaks zu entzerren, ist es wichtig, dass der Therapiebeginn flexibel gestaltet werden kann. Dies sei mit vielen neuen Präparaten möglich. „Wir können präsaisonal beginnen, intrasaisonal, perennial und prä-/co-saisonal“, bestätigte Horn. "Wenn der Patient sich in der Praxis vorstellt, dann wird er nach Möglichkeit sofort behandelt."

Horn verwendet subkutan z. B. das immunologisch verstärkte Depotpräparat Avanz ${ }^{\circledR}$ von ALK-Abelló. Es sei gegenüber dem Vorgänger ALK SQ ${ }^{\circledR}$ bei gleicher Immunogenität und Sicherheit schneller aufdosierbar und durch das optimierte Verhältnis von Allergen und Adjuvans komme es noch seltener zur Gra- nulombildung. Durch das lange Erhaltungsintervall von bis zu acht Wochen ließen sich auch Urlaubszeiten gut überbrücken.

Weiter berichtete Horn über eigene Erfahrungen mit der Gräser-Sublingualtablette Grazax ${ }^{\circledR}$. Ihre Wirksamkeit sei mit hoher Evidenz nachgewiesen und halte bis zu zwei Jahre über das Therapieende hinaus an. Die Therapie könne damit sehr flexibel beginnen wie auch prä-/co-saisonal erfolgen. Grazax ${ }^{\circledR}$ habe keine lange Lieferzeit, sondern stehe als Fertigprodukt in der Apotheke sofort zur Verfügung. Die schnelle Rezeptierung sei gut in den Praxisalltag integrierbar.

Horn fasste zusammen: „Der richtige Therapiemix ist für mich die $\mathrm{Zu}$ kunftschance der Allergologie." Seine persönliche Formel für die Praxis lautet: „Zugelassene Präparate mit hoher Evidenz ... und bei mir gibt es keine präsaisonale Therapie, sondern ich versuche die Belastung übers ganze Jahr konstant zu halten." Dr. Dana Weiß

Symposium „Perspektiven im Spannungsfeld Arzt , Patient und Kostenträger" im Rahmen des 6. Deutschen Allergiekongresses. Wiesbaden, 10. September 2011. Veranstalter: ALK-Abelló, Wedel

\section{Wirkung der Immuntherapie unterschätzt - aber nicht mehr lang}

D ie übliche Pollenflug-basierte Festlegung des Auswertungszeitraums in klinischen Studien zur Wirksamkeit der spezifischen Immuntherapie (SIT) bei allergischer Rhinitis könnte das Ergebnis verzerren. Wie Prof. Dr. KarlChristian Bergmann, Berlin, beim 6 . Deutschen Allergiekongress in Wiesbaden erklärte, werden allergische Reaktionen nicht bei allen Betroffenen durch die gleiche Zahl von Pollen ausgelöst, sondern jede Person hat einen eigenen Schwellenwert. Daneben beeinflussen Faktoren wie Alter, Lebensweise und Infekte die Reaktionsstärke.

Prof. Dr. Ralph Mösges hob hervor, dass die Zahl der Pollen, die in Fallen gesammelt werden, nicht die individuelle Allergenbelastung des Patienten wi- derspiegele - schon allein aufgrund der variierenden Distanz seines Aufenthaltsorts zur Pollenfalle. Wird der Auswertungszeitraum statt am Pollenflug (PFA) an den stärksten Beschwerden in der Plazebogruppe (PGA) festgemacht, so erhöhe sich die statistische Trennschärfe zwischen den Studiengruppen und die Symptomlinderung durch die SIT werde genauer erfasst. Beispielhaft gezeigt wurde dies anhand der retrospektiven Auswertung der G301-Studie mit 1.028 doppelblind-randomisierten Probanden aus USA, Kanada und Österreich.

Der Verumgruppe war präsaisonal das Präparat Pollinex ${ }^{\circledR}$ Quattro mit Allergoiden plus dem Adjuvans Monophosphoryl-Lipid A $\left(\mathrm{MPL}^{\circledR}\right)$ injiziert worden, der Plazebogruppe ein tyrosin- haltiges Trägermedium. Ein elektronisches Tagebuch fragte über zehn Wochen hinweg täglich die Symptome an Augen und Nase sowie die Bedarfsmedikation ab. Der kombinierte SymptomMedikations-Score für die Verumgruppe verbesserte sich signifikant um 26,9\% berechnet auf PFA-Basis und sogar um $30,3 \%$ auf PGA-Basis gegenüber der Plazebogruppe. Besonders profitierten von der Therapie europäische Patienten aus Zentren mit hoher Pollenbelastung bzw. starken Beschwerden. Hier besserte sich der Score der Verumgruppe gegenüber der Plazebogruppe um 38,3\% berechnet auf PFA-Basis bzw. 46,3\% auf PGA-Basis. Die spezifische Immuntherapie mit Allergoiden plus Adjuvans subkutan injiziert ist demnach noch wirksamer als bisher gedacht.

red

Nach Informationen von Bencard, München 